Relations industrielles

Industrial Relations

\title{
Le bilan des relations patronales-ouvrières
}

\section{Roger Vézina}

Volume 5, numéro 4, janvier 1950

URI : https://id.erudit.org/iderudit/1023314ar

DOI : https://doi.org/10.7202/1023314ar

Aller au sommaire du numéro

Éditeur(s)

Département des relations industrielles de l'Université Laval

ISSN

0034-379X (imprimé)

1703-8138 (numérique)

Découvrir la revue

Citer cet article

Vézina, R. (1950). Le bilan des relations patronales-ouvrières. Relations

industrielles / Industrial Relations, 5(4), 33-35. https://doi.org/10.7202/1023314ar

Tous droits réservés @ Département des relations industrielles de l’Université Laval, 1950
Ce document est protégé par la loi sur le droit d'auteur. L’utilisation des services d'Érudit (y compris la reproduction) est assujettie à sa politique d'utilisation que vous pouvez consulter en ligne.

https://apropos.erudit.org/fr/usagers/politique-dutilisation/ 


\title{
UN BILAN DES RELATIONS PATRONALES-OUVRIĖRES
}

\author{
ROGER VÉZINA
}

\section{Relations patronales- ouvrières}

La Chambre de commerce du Canada vient de publier les résultats d'une enquète ${ }^{1}$ conduite auprès de quelque 1,300 entreprises canadiennes ayant à leur emploi 10 salariés et plus. Divisé en deux parties, le questionnaire auquel avaient à répondre les patrons couvrait le vaste terrain des relations patronales-ouvrières d'une part, et, d'autre part, des mesures de sécurité sociale proprement dites. Les résultats de l'enquête démontrent que si les relations patronales-ouvrières en sont encore au stage de l'enfance, par contre les mesures de sécurité sociale ont accompli un pas de géant depuis quelques années. Dans la majorité des entreprises consultées existent déjà des. plans d'assurance-groupe, de pensions de retraite et d'indemnités ou d'hospitalisation en cas de maladie. Les quatre tableaux qui illustrent cet article, gracieusement mis à notre dis-

(1) Survey Results Employer-Employee Relations 1949. - Issued by The Canadian Chamber of Commerce, 530, Board of Trade Building, Montreal. - 75 cts per copy. position par la revue officielle de la Chambre de commerce du Canada, «Canadian Business », sont très révélateurs de cette dernière prétention.

La première partie du questionnaire, portant sur les relations patronales-ouvrières, ne comptait pas moins de dix-huit questions susceptibles d'illustrer à la fois les nombreuses façons d'établir et entretenir de semblables relations et le degré de popularité de chacune d'elles dans l'entreprise. Compilées dans une bonne soixantaine de tableaux statistiques, les réponses reçues ont été partagées en deux groupes génériques: le premier illustrant le total des réponses données par les 1,309 entreprises consultées, elles-mêmes divisées en cinq groupes numériques de 10 à 99 , 100 à 249,250 à 499,500 à 999 , 1,000 à 4,999 et de 5,000 employés et plus, et, le deuxième illustrant le total des réponses données par les seules entreprises manufacturières, 730 au total, elles-mêmes divisées en cinq groupes numériques de 10 à 99 employés, etc. Il va sans dire qu'il nous est absolument impossible de disséquer dans le cadre de ce bref article des tableaux statistiques qui ne couvrent pas moins d'une bonne cinquantaine de pages. Nous nous en tiendrons donc à quelques résultats d'ensemble capables d'illustrer le degré de popularité de telle ou telle politique de relations patronaiesouvrières, telle ou telle mesure de sécurité sociale.

Ainsi, les réponses données démontrent que:

1. Dans 456 entreprises, dont 321 manufacturières, sur un total de 1,309 , une ou plusieurs personnes s'occupent spécialement du problème des relations patronales-ouvrières. Exprimés en pourcentages les totaux nous donnent $35 \%$ de toutes les entreprises consultées et $44 \%$ des seules entreprises manufacturières;

2. A date, seulement 167 entreprises, ou $13 \%$ du total, ont déjà consulté l'opinion de leurs employés;

3. 355 ou $27 \%$ des entreprises publient un manuel de la compagnie destiné aux employés. Les sujets abordés dans ces manuels por-

\section{Technigues des relations de trawail}

Le Rapport du quatrième Congrès des relations industrielles de Laval (1949) contenant le texte des conférences et des délibérations vient de paraître. On peut s'en procurer des exemplaires au Département des relations industrielles, Faculté des sciences sociales, 2, rue de l'Université, Québec. Prix: $\$ 1.50$ (franco).

Sujets traités: Conciliation et arbitrage (Me Louis-Philippe Pigeon); Organisation patronale (Louis Bilodeau); Organisation ouvrière (Jean Marchand); Négociation de la convention collective - aspect patronal (Hector Cimon); Négociation de la convention collective - aspect ouvrier (Rémi Duquette); Application de la convention collective et administration du personnel - aspect patronal (Lawrence A. Lyons); Application de la convention collective et délégués du personnel - aspect ouvrier (René Gosselin). 
ASSURANCE-GROUPE

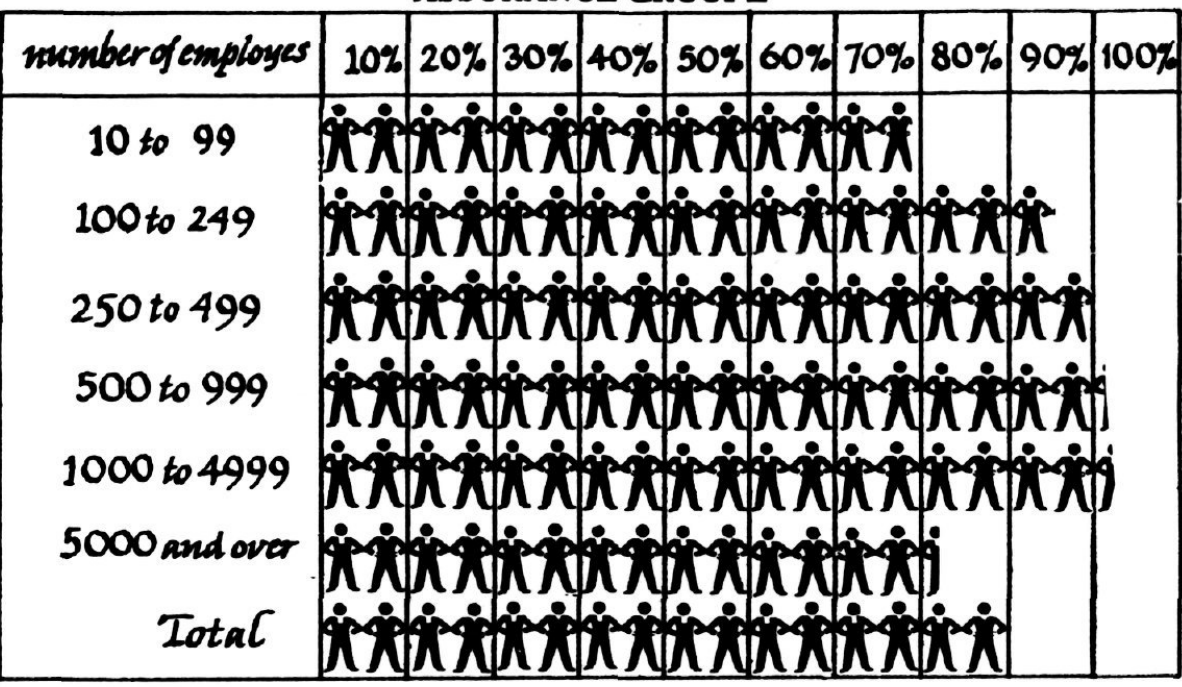

tent soit sur les règlements, soit sur la politique et les buts, soit encore sur l'histoire de la compagnie ou autres sujets;

4. 611 entreprises, ou $47 \%$ du total, ont une politique d'initiation pour leurs nouveaux employés: présentation des administrateurs, visite de l'entreprise, explication de ses rouages, etc;

5. Sous la forme de bulletins, lettres circulaires, etc., imprimés ou stencilés, mensuels, hebdomadaires, etc., 355 entreprises, ou $27 \%$, destinent des publications à leurs employés;

6. 734 entreprises, ou $56 \%$, convoquent leurs employés en «meeting 》 général ou par petits groupes, ou des deux façons à la fois;

7. 981 , ou $75 \%$ du total, organisent des assemblées pour les contremaîtres ou autres directeurs du personnel;

8. 177 , ou $13 \%$, ont un programme d'entraînement pour les administrateurs de l'entreprise;

9. 930 , ou $25 \%$, entraînent leurs contremaîtres;
10. 421, ou $32 \%$, entraînent leurs employés;

11. 450 , ou $34 \%$, s'occupent de faire visiter leurs entreprises, tenir ou participer à des expositions de leurs produits. Exclues les entreprises qui, pour une raison ou une autre, ne peuvent donner suite à un tel programme, le pourcentage s'élève à $59 \%$;

12. Régulièrement ou occasionnellement, par lettres, bulletins ou autrement, 457 entreprises, ou $35 \%$, communiquent d'une façon quelcon-
RETRAITE que avec leur employés à domicile;

13. $86 \%$ des entreprises affichent périodiquement des bulletins dans des endroits stratégiques;

14. $13 \%$ des entreprises circularisent le rapport annuel de la compagnie auprès de leurs employés et $4.5 \%$ ont une édition spéciale de ce rapport pour leurs employés;

15. 520 , ou $40 \%$, ont adopté un système quelconque de partage des profits, sous la forme de bonus ou autrement;

16. 332 , ou $25 \%$, ont un système de vérification du rendement du personnel;

17. 279 , ou $21 \%$, s'occupent d'évaluer et récompenser le mérite;

18. 562 , ou $43 \%$, ont adopté une politique définie de promotions;

19. 306 , ou $23 \%$, s'occupent de relations extérieures (avec le public) dans les journaux locaux.

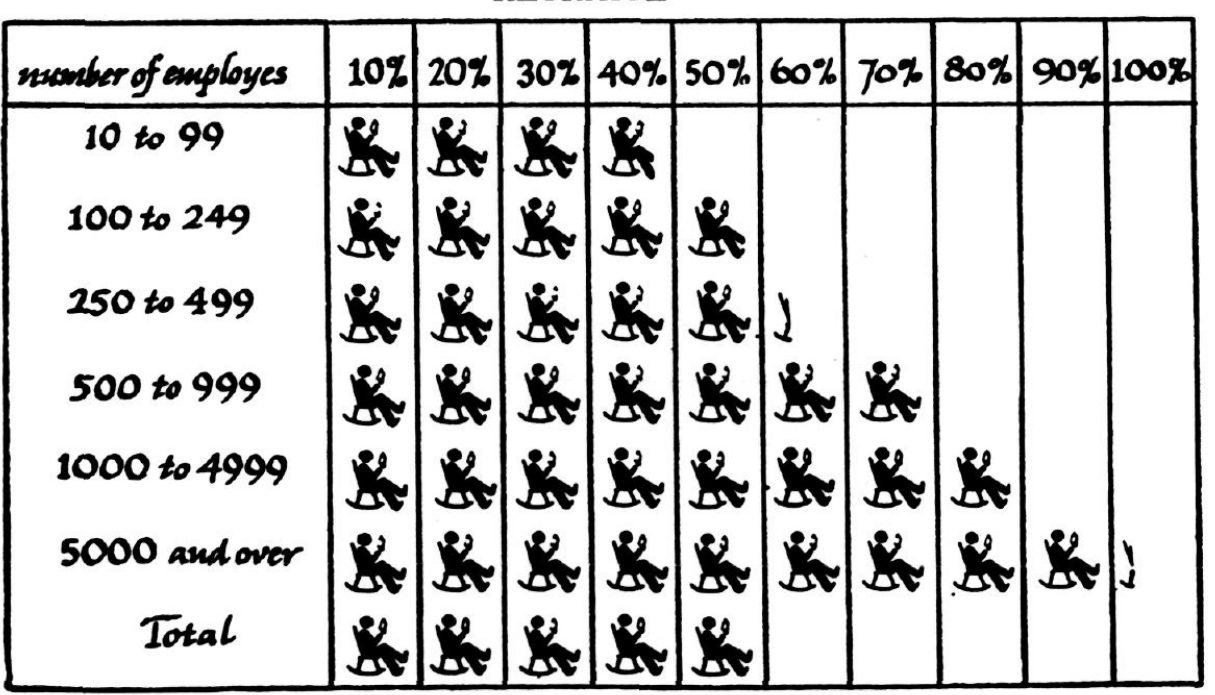


Sécurité sociale

1. 1,042 entreprises sur un total de 1,309 , soit $80 \%$, et 610 entreprises spécifiquement manufacturières sur un total de 730 , soit $83.5 \%$, ont adopté un plan quelconque d'assurance-groupe. Sur le grand total, 810 ont opté pour un plan contributoire, 147 pour un plan non-contributoire et 62 s'abstiennent de préciser;

2. 657 entreprises sur 1,309, soit $50 \%$ du total, et 365 entreprises spécifiquement manufacturières sur 730 , soit $50 \%$ également du total, ont adopté un système de pension de retraite. Sur le grand total, 533 sont contributoires et 101 non-contributoires. Une vingtaine d'entreprises s'abstiennent de préciser;

3. Sur les 635 entreprises qui n'ont pas de système de pension de retraite, 166 s'occupent d'aider d'une façon quelconque leurs employés retraités, 346 ne font rien et 123 n'apportent aucune précision;

4. 988 entreprises, ou $75.5 \%$ du total, font profiter leurs
hOSPITALISATION

\begin{tabular}{|c|c|c|c|c|c|c|c|c|c|c|}
\hline number of employes & $10 \%$ & $20 \%$ & $30 \%$ & $40 \%$ & $50 \%$ & $60 \%$ & $70 \%$ & $80 \%$ & $90 \%$ & $100 \%$ \\
\hline 10 to 99 & & & & & & & & & & \\
\hline 100 to 249 & & & & & & & & & & \\
\hline 250 to 499 & & & & & & & & & & 3 \\
\hline 500 to 999 & & & & & & & & & & \\
\hline 1000 to 4999 & & & & & & & & & & 7 \\
\hline 5000 and over & & & & & & & & & & \\
\hline
\end{tabular}

employés d'indemnités en cas d'absence pour cause de maladie. Dans 426 entreprises les indemnités sont établies sur une base contributoire, dans 380 , sur la base non-contributoire. Pour le reste on n'apporte aucune précision;

5. 1,122 établissements, ou $85.5 \%$ du total, pourvoient à l'hospitalisation de leurs employés. Sur ce total, 857 ont un plan d'hospitalisation contributoire, 166, un plan non-contributoire et 99 ne précisent pas;

6. 435 entreprises, ou $33 \%$ du total, ont élaboré un programme récréatif pour leurs employés;

7. 372 , ou $28.5 \%$ du total, ont un service médical doté d'un personnel spécialisé;

8. 390 , ou $30 \%$ du total, font subir un examen médical préalable aux candidats à un emploi;

9. 299 , ou $22 \%$ des entreprises consultées, font subir à leurs employés un examen médical périodique;

INDEMNITES EN CAS DE MALADIE

\begin{tabular}{|c|c|c|c|c|c|c|c|c|c|c|}
\hline ber of employes & $10 \%$ & $20 \%$ & $30 \%$ & $40 \%$ & $50 \%$ & $60 \%$ & $70 \%$ & $80 \%$ & $90 \%$ & $100 \%$ \\
\hline 10 to 99 & & & & & & & & & & \\
\hline 100 to 249 & & & & & & & & & & \\
\hline 250 to 499 & & & & & & & & & H & \\
\hline 500 to 999 & & & & & & & & & & \\
\hline 1000 to 4999 & & & & & & & & & & $\beta$ \\
\hline 5000 and over & & & & & & & & & Ry & P \\
\hline Total & & & & & & & & & & \\
\hline
\end{tabular}

10. 396 , ou $30 \%$ du total, opèrent des restaurants pour le personnel. Dans $34.5 \%$ les restaurants sont opérés à prix coûtant, dans $37 \%$ audessous du prix coûtant, $16 \%$ au-dessus du prix cốtant. Le $12 \%$ qui reste ne précise pas;

11. Enfin, à la question: « Vos employés horaires sont-ils membres d'un syndicat ? 》, les 730 entreprises manufacturières consultées ont répondu comme suit: oui, 416 entreprises ou $56 \%$ du total; non, 274 ou $38 \%$; partiellement, 34 ou 5\%; aucune précision, 6 ou $1 \%$. 